\title{
Cloud Formation in the Plumes of Solar Chimney Power Generation Facilities: A Modeling Study
}

\author{
Timothy M. VanReken \\ Laboratory for Atmospheric Research, Department of Civil \& Environmental Engineering, \\ Washington State University, PO Box 642910, Pullman, Washington, 99164-2910, USA \\ e-mail: vanreken@wsu.edu \\ Athanasios Nenes \\ Schools of Earth \& Atmospheric Sciences and Chemical \& Biomolecular Engineering, Georgia \\ Institute of Technology, 311 Ferst Drive, Atlanta , Georgia 30332-0340, USA
}

\begin{abstract}
The solar chimney power facility has the potential to become a valuable technology for renewable energy production. Its financial viability depends on a thorough understanding of the processes affecting its performance, particularly because of the large startup costs associated with facility design and construction. This paper describes the potential impacts on plant capacity resulting from cloud formation within or downwind of the solar chimney. Several proposed modifications to the basic concept of the solar chimney power facility have the potential to cause significant additions of water vapor to the air passing through the collector. As the air continues up through and out of the chimney, this excess water can condense to form cloud. This possibility is explored using a cloud parcel model initialized to simulate the range of expected operating conditions for a proposed solar chimney facility in southwestern Australia. A range of temperatures and updraft velocities are simulated for each of four seasonal representations and three levels of water vapor enhancement. Both adiabatic environments and the effects of entrainment are considered. The results indicate that for very high levels of water vapor enhancement cloud formation within the chimney is likely; at more moderate levels of water vapor enhancement the likelihood of plume formation is difficult to fully assess as the results depend strongly on the choice of entrainment rate. Finally, the impacts of these outcomes on facility capacity are estimated.
\end{abstract}




\section{Introduction}

In recent years electricity costs have consistently increased as a result of rising fossil fuel demand and uncertainties in future supplies. In addition to the direct costs associated with fossil fuel power generation are the indirect costs realized through increased atmospheric pollution and the threat of global climate change. At the same time, there exists an ever increasing demand for electrical power, particularly in remote developing regions. Combined, these factors are strong motivations for the continued improvement of renewable power production technologies. Ideally, renewable energy power plants convert energy from naturally occurring thermal or kinetic sources into electricity in an economically viable manner, while minimizing negative impacts on the environment.

One technology for converting solar energy into electricity that has shown promise in recent years is the so-called solar chimney (or solar tower) power plant. A good overview of the technology has been provided by Schlaich et al. [1], including the theoretical principles governing its design. The concept of the solar chimney is straightforward and consists of three main components (Figure 1): a solar air collector, one or more air turbines, and the chimney itself. The solar air collector is a large, roughly circular, greenhouse-like structure that gently slopes toward its center. Air enters at the outer edge of the collector, and as it warms buoyancy causes it to move upward and inward toward the collector's center. When the air has reached the center its temperature has increased substantially, at which point it enters the tower itself. The tower functions as the main thermal engine in the power plant; the available power for electrical conversion depends largely on the driving potential pressure, which in turn depends primarily on the height of the tower and on the temperature increase. The actual power output is affected by the efficiency of the turbines, which are placed between the solar collector and the chimney and convert a fraction of the available kinetic energy into electricity. Thus, the power capacity of a solar chimney facility can be expressed as:

$$
P_{\text {tot }}=\left(G \cdot A_{\text {coll }}\right) \cdot\left(\eta_{\text {coll }} \cdot \eta_{\text {chim }} \cdot \eta_{\text {turb }}\right)
$$

$P_{\text {tot }}$ is the power generated by the facility. $G$ is the incoming solar energy flux and $A_{\text {coll }}$ is the area of the solar collector, so $\left(G \cdot A_{\text {coll }}\right)$ defines the power input to the system in the form of incoming solar radiation. The collector efficiency, $\eta_{\text {coll }}$, describes how efficiently this thermal energy is converted to potential energy in the form of the heated, buoyant air at the base of the chimney. $\eta_{\text {chim }}$, the chimney efficiency, describes how efficiently, in the absence of any turbine activity, this potential energy would be converted to kinetic energy in the form of air moving up the chimney. 
$\eta_{\text {turb }}$ is the turbine efficiency, and expresses how effectively the kinetic energy of the air is converted to electricity. The overall plant capacity is strongly dependent on the size of the facility, both in terms of the collector area (through its effect on the energy input into the system), and the tower height (through its effect on the chimney efficiency).

This general concept for the solar chimney power plant is several decades old but there has been increased interest in the technology more recently. A functioning prototype of moderate capacity operated in Manzanares, Spain from 1982 to 1989 [2,3]. This facility's collector had an mean radius of $122 \mathrm{~m}$ and its tower was $195 \mathrm{~m}$ high and $10.2 \mathrm{~m}$ across; the nominal capacity was 50 kW [1]. Experimental data from the Manzanares facility have been used extensively to develop and validate generalized design models for solar chimney facilities [4,5]. More importantly, the successful operation of the Manzanares facility has resulted in plans for additional, larger operational solar tower power plants, at locations including both northwestern China [6] and southeastern Australia [1]. The planned facility in China will include a tower of essentially the same height and diameter as the Manzanares facility, though a larger collector and other design improvements are expected to increase generating capacity by a factor of approximately two to four, to $110-190 \mathrm{~kW}$ [6]. The proposed Australian plant, when completed, will be much larger: Schlaich et al. [1] reported that the design was for a $200 \mathrm{MW}$ capacity facility, requiring a $1000 \mathrm{~m}$ tower height and a $7000 \mathrm{~m}$ collector diameter. However, a recent report from the company developing the facility indicates that the design has been scaled back to a 50 MW capacity, primarily due to concerns about the proposed tower height, which would have been the world's tallest manmade structure [7].

One factor affecting the scale-up of solar chimney power facilities is the impact that water vapor has within the system. Most studies have ignored the presence of water vapor altogether, implicitly assuming that the impact is negligible and that while the engineering challenges of scale-up are significant, the thermodynamics of the problem remain essentially the same. The potential impact of water vapor was discussed by Kröger and Blaine [8], who showed that moist air could improve plant performance. A more recent study by Ninic [9] also addressed this effect of water vapor on system efficiency, demonstrating that increasing the humidity at the collector inlet to the degree possible would maximize the potential energy of the air entering the base of the chimney. Ninic ultimately concluded that evaporating water in the collector would not be a cost effective means of increasing plant capacity, but also noted that increasing water vapor content of the air entering the collector would be beneficial. Other design modifications proposed to improve the economic viability of solar tower facilities also have the potential to significantly 
increase the water vapor content of air moving through the system. In one, described by Schlaich et al. [1], water filled black tubes would be incorporated into the collector. These devices would store heat during the day and release it overnight so that the plant could produce electricity more consistently; some minor evaporative losses would almost certainly result, though Schlaich et al. explicitly discount this possibility. Another modification would be to use the area under the solar collector for agriculture, as has been proposed by Dai et al. [6]. Evaporative losses from irrigation would be another, potentially far larger, source of water vapor in the system.

These modifications are proposed as mechanisms to improve the performance and/or the economic viability of solar chimney power generation facilities. However, there is one impact of enhanced water vapor that has yet to be considered in detail and may be significant - the potential effect of condensable water vapor in the air rising through and exiting the solar chimney. When a moist air mass rises, the temperature decreases, which causes the relative humidity to increase. Once the saturation temperature is reached, water vapor will begin to condense onto any available surface, including any particles that are present in the air mass; this is the same process by which clouds form. Kröger and Blaine [8] showed that condensation could occur within a solar chimney, but did not explore the implications of the phenomenon.

The prospect of increased water vapor greatly increases the likelihood that water condensation will occur in the air mass as it proceeds up through and out of the solar tower. This study explores these potential effects and how they may affect the performance of a solar chimney power facility. Two effects in particular will be explored: the potential for condensation to form within the chimney (including even the possibility of precipitation), and the effect of any plume formation and propagation after the air mass leaves the chimney. The likelihood of these effects will be evaluated using a cloud parcel model. After initiating the simulations with conditions consistent with solar chimney power plant operation, the results will indicate the height where condensation begins, and will provide temperature, droplet size distribution, and liquid water profiles as a function of height. First, a series of adiabatic simulations is presented to characterize plume behavior across a range of temperature increases and tower updraft velocities, for relative humidities consistent with either moderate and significant water vapor enhancement in the collector region. A more limited set of studies with no water vapor enhancement in the profile are also included for comparison. Then, the results from the adiabatic simulations are used to inform an additional series of parcel simulations that include entrainment (i.e., the mixing of ambient air into the parcel) after the parcel has left the chimney. These last simulations are used to evaluate what potential impacts water condensation might have on overall solar chimney 
power plant performance. For convenience, the simulations and analyses will largely assume a hypothetical plant similar to the proposed Australian facility described by Schlaich et al. [1].

\section{Methods}

\subsection{Cloud parcel model}

The cloud parcel model used in this study was developed as a Fortran code by Nenes et al. [10] based on the equations of Pruppacher and Klett [11] and Seinfeld and Pandis [12]. This model has been used previously primarily to explore the potential for kinetic effects to impact cloud droplet formation and thereby cloud albedo [13-15]. Cloud parcel models follow a moist air mass as it rises; they are usually adiabatic models, conserving both heat and moisture. As the parcel rises, the temperature decreases, and the water saturation ratio increases accordingly. Contained in the air mass is an aerosol population with prescribed size distribution and chemical composition; as the air mass approaches and eventually surpasses its saturation point, cloud droplets form in accordance with droplet activation (Köhler) theory [12]. Additional details can be found in the study by Nenes et al. [10].

Initializing a cloud parcel model requires specifying the characteristics of the parcel to be tracked, the aerosol population within the parcel, and the conditions of the ambient atmosphere (if the simulation is non-adiabatic). The parcel is defined by its initial pressure, temperature, relative humidity, liquid water content, and updraft velocity. The aerosol population within the parcel is described as a set of lognormal particle size distributions, each of which can be split into separate internally mixed soluble, partially soluble, and insoluble fractions of different densities and chemical characteristics. For non-adiabatic simulations, the atmosphere surrounding the parcel is also specified with respect to its temperature, its vertical temperature profile (the lapse rate), and its vertical water vapor profile. Finally, the type of simulation and its duration are defined.

In the current study, the assumption that a parcel is adiabatic is presumed to be reliable while it remains in the chimney, but not once the parcel exits the chimney. To more realistically portray a cloud parcel as it moves through and out of a solar chimney, the cloud parcel model has been modified to include entrainment only after the parcel exits the chimney. At the start of the simulation, when the parcel would be inside the chimney, the model still treats the parcel adiabatically with a constant updraft velocity. Once the parcel exits the chimney, however, it is no longer realistic to treat it as isolated; it would undoubtedly start to mix with the surrounding air. The modified model addresses this eventuality by considering entrainment processes only 
after a specified simulation time has been reached; because of the simulation's constant updraft velocity, this transition time can be chosen to correspond to the assumed chimney height.

\subsection{Initialization conditions}

The initialization conditions were chosen to represent the ranges of environmental conditions that might be expected for the solar chimney power plant currently being developed by EnviroMission, Ltd., in southwestern Australia. Details informing the choice of initial conditions were drawn from descriptions of this project found in the study by Schlaich et al. [1] and on the EnviroMission website (www.enviromission.com.au). The facility is to be built in a remote region in southeastern Australia, and is to operate year-round.

The ambient temperature $(T)$ and relative humidity $(R H)$ for the simulations were chosen to reflect this location. To explore the potential seasonal variability in the results, simulations were performed for each of four representative months: January, April, July, and October. Representative values for $T$ and $R H$ were selected using the meteorological statistics for Wentworth, New South Wales, a small city near the site proposed for the power plant; these statistics may be found at the Australian Bureau of Meteorology website (http://www.bom.gov.au/climate/averages/tables/cw_047053.shtml). For each of the months, two sets of simulations were performed, to represent two possible humidity states as the parcel enters the base of the chimney. The "LoRH" cases represent situations in which the relative humidities at the base of the solar chimney are the same as the ambient relative humidities (i.e., enough water vapor is added to the parcels in the solar air collector such that the relative humidities remain constant). The "HiRH" cases represent far more substantial additions of water vapor to the parcel as it passes through the solar air collector; in these cases the relative humidities at the base of the chimney are always $80 \%$. A small set of "NoEnhance" simulations were also performed for a baseline comparison, wherein no water vapor was assumed to be added to the parcel as it passed through the solar collector. A summary of these seasonal cases is presented in Table 1 .

The seasonally representative temperatures presented in Table 1 are ambient temperatures; that is, they are the temperature of the parcel when it enters the solar air collector. As the parcel moves through the solar collector, the temperature increases by $\Delta T$, and the parcel then accelerates up through the solar chimney to its stable vertical velocity, $w$. Schlaich et al. [1] indicate that for normal operating conditions at the initially proposed Australian facility, $\Delta T$ and $w$ would be $\sim 30$ $\mathrm{K}$ and $\sim 15 \mathrm{~m} \mathrm{~s}^{-1}$, respectively. For this study, however, it was desirable to examine the potential 
effects of water vapor in the system across a broader range of performance criteria. Thus five values of $\Delta T(15,25,30,35$, and $40 \mathrm{~K})$ and five values of $w\left(1,5,10,15\right.$, and $\left.20 \mathrm{~m} \mathrm{~s}^{-1}\right)$ were chosen; adiabatic simulations were performed for each combination of $w$ and $\Delta T$, for each of the eight seasonal representations. While some of the specific combinations of $\Delta T$ and $w$ are physically improbable, all were included here to highlight the trends as the two parameters are varied. The "NoEnhance" baseline simulations were performed for $\Delta T=30 \mathrm{~K}$ and $w=15 \mathrm{~m} \mathrm{~s}^{-1}$ only. All of these parameters are presented in Table 2, along with the other initialization parameters required for the parcel model simulations.

The entraining parcel model requires two operating parameters in addition to those described above. These are the entrainment rate, and the transition time (i.e., the time after which entrainment is allowed to occur). The transition time for each entrainment simulation is chosen to correspond to the hypothetical $1000 \mathrm{~m}$ chimney height (for a constant updraft velocity, simulation time and parcel height correspond directly). Estimating the entrainment rate is more difficult; its value depends strongly on the local meteorological conditions, is highly variable, and difficult to measure. To bracket the range of possible values, this study includes simulations where the entrainment rate is $30 \% \mathrm{~km}^{-1}$ (chosen to match the average values calculated by Jensen and Del Genio [16] for cumulus congestus cloud) and a second set with a much higher entrainment rate of $30 \%$ per $100 \mathrm{~m}$. (Conceptually, a $30 \% \mathrm{~km}^{-1}$ entrainment rate implies that, for every $1000 \mathrm{~m}$ increase in height, $30 \%$ of the air in the parcel is exchanged with the surrounding ambient air.) All of the "LoRH" and "NoEnhance" cases were simulated using the modified model; the "HiRH" simulations were not repeated, for reasons to be discussed below.

The final set of initialization parameters to be determined are those specifying the aerosol population. Jaenicke [17] reported typical aerosol size distributions for a variety of environments as linear combinations of lognormal modes. In accordance with the remote site of the proposed Australian solar chimney facility, Jaenicke's suggested distributions for remote continental sites were used in this study. Each mode of the aerosol is assumed to be composed of ammonium sulfate internally mixed with some insoluble fraction; the insoluble fractions were estimated using the same reasoning as Lance et al. [15]. The parameters used to define the aerosol population are presented in Table 3; $D_{p}$ is the geometric mean diameter for each mode, $N$ is the particle concentration in the mode, and $\sigma$ is the geometric standard deviation. 


\section{Adiabatic cloud parcel simulations}

The first set of cloud parcel simulations undertaken for this study were adiabatic. Adiabatic simulations are idealized, in that they are based on the assumption that there is no exchange of heat or water vapor between the parcel and the surrounding air. Adiabatic simulations are also substantially less expensive computationally, and generally set an upper limit on the magnitude of a given effect. The tradeoff for these advantages is that adiabatic simulations are not entirely realistic. In this case the assumption is probably realistic when the parcel would be inside the chimney, but much less so afterwards. A total of 404 adiabatic parcel simulations were performed during the study. Four were baseline "NoEnhance" cases, one each for the seasonally representative months; the other four hundred included each possible combination of $\Delta T$ and $w$ for each of the eight seasonally representative cases described in Section 2.2.

Vertical profiles from a typical adiabatic simulation are presented in Figure 2. These profiles are from the October "LoRH" simulation, with $\Delta T=30 \mathrm{~K}$ and $w=15 \mathrm{~m} \mathrm{~s}^{-1}$. As the parcel rises at the start of the simulation (i.e., as the height increases), the temperature decreases (Fig. 2a), and the saturation ratio increases (Fig. 2b; note that the supersaturation is the saturation ratio minus one). This initial increase in saturation ratio is caused only by the change in saturation vapor pressure due to the temperature decrease. The liquid water mixing ratio remains at zero during the initial stages of the simulation. The water vapor mixing ratio is also essentially constant, though there is a minor numerical drift that is a result of the way the model initializes the equilibrium state of the aerosol. These mixing ratio trends continue until the parcel reaches its saturation point.

When the parcel surpasses its saturation point (at $\sim 2700 \mathrm{~m}$ in Fig. 2), water vapor begins to condense onto the available particles; this onset of condensation conventionally defines the base of the cloud. The condensation process is not instantaneous. Rather, its rate is driven by the magnitude of the supersaturation (i.e., the amount by which the saturation vapor pressure is surpassed). Thus, a significant supersaturation can be observed in the rising parcel at cloud base before the conversion of water from the vapor to the liquid phase causes the saturation ratio to begin to decrease. In the case presented in Fig. 2, the maximum supersaturation is $\sim 0.70 \%$. As the parcel continues upward from the supersaturation maximum, water continues to condense from the vapor phase, and the saturation ratio regresses toward its equilibrium value of 1.0. In these adiabatic simulations, the regression is asymptotic; the cooling parcel continuously creates excess water vapor available for condensation. In reality, entrainment of external air would eventually reduce the humidity to below the saturation point. 
The first key question regarding the potential influence of enhanced water vapor on a solar chimney power plant is whether the condensation process could begin while the parcel is still in the chimney. This question is readily addressed by examining the results of the adiabatic simulations (Figures 3 and 4 for the "LoRH" and "HiRH" conditions, respectively). The results demonstrate that the cloud base heights are independent of the updraft velocity; this is characteristic of the adiabatic condition. For each of seasonal representations, there is a dependence of the cloud base height on the on the temperature increase. When there is moderate water vapor enhancement (the "LoRH" cases), cloud base occurs between 1300 and $3800 \mathrm{~m}$. However, the dependence on the amount of water vapor enhancement is much stronger. At larger water vapor enhancements (the "HiRH" cases), the saturation point is reached at much lower heights, between 500 and $700 \mathrm{~m}$. For the cases where there is no enhancement to the water vapor mixing ratio, condensation does not occur below $5000 \mathrm{~m}$ (not shown).

The dependence of the saturation height on the water vapor mixing ratio can also be seen in the interseasonal variability. For both the no enhancement and moderate enhancement cases, the saturation height varies according to the ambient relative humidity, with the lowest cloud bases occurring during the wettest seasons and vice versa. The interseasonal variability can be substantial - in the "LoRH" simulations cloud base was twice as high in the driest season (January) compared to the wettest (July). This is directly due to the variation in ambient relative humidity; in the "HiRH" simulations, there was almost no interseasonal variability, because in those cases the assumed relative humidity was independent of the season.

In addition to the cloud base height, Figs. 3 and 4 also present the maximum supersaturations and liquid water contents for the adiabatic cloud parcel simulations. In contrast to the cloud base height, the maximum supersaturation is a strong function of the updraft velocity and a weaker function of the temperature increase. In all cases the maximum supersaturation occurs a short distance above the cloud base height. The strong dependence on the updraft velocity arises because condensation of water onto the aerosol population is a kinetic process. When the air is rising more rapidly, a greater distance beyond the saturation level can be reached before the rate of condensation "catches up" to the rate of saturation level increase. Thus higher updrafts will lead to higher maximum supersaturations. The maximum liquid water content for these adiabatic simulations are less meaningful. As expected, these results exhibit very small dependences on temperature and updraft velocity, but in each case the maximum liquid water content occurs at the end of the simulation, an unrealistic outcome of the adiabatic condition. 
From the cloud base heights in Figs. 3 and 4, it is clear that condensation will not occur in the hypothetical $1000 \mathrm{~m}$ chimney unless there is substantial water vapor enhancement. For the cases considered here, none of the simulations with moderate water vapor enhancement (the "LoRH" cases) produced a cloud base below $1300 \mathrm{~m}$, and all of the "HiRH" cases, with greater water vapor enhancement, produced liquid water at $700 \mathrm{~m}$ or below. The next key question is determining how cloud development proceeds for the "HiRH" cases from cloud base to the point where the parcel exits the chimney and particularly, whether precipitation might form before the parcel leaves the chimney.

Addressing this question is accomplished by examining the evolution of the cloud droplet distribution in the rising parcels. As the air parcels surpass their saturation point, the excess water condenses on the available particles, causing them to activate and become cloud droplets. These newly formed droplets rapidly grow to many times their initial size. This process is simulated in the cloud parcel model; an example of the evolution of the cloud droplet distribution is presented in Figure 5. At the start of the simulation in the example, nearly all particles have diameters less than one micron, and the size distribution changes very little until the saturation point is reached. This occurs at a height of $\sim 600 \mathrm{~m}$ in Figure 5. At that height much of the aerosol forms droplets, or "activates", and the mode diameter of the droplet size distribution rapidly grows to greater than five micrometers, and is greater than nine micrometers when the chimney top height of $1000 \mathrm{~m}$ is reached. At chimney top, some of the droplets have already grown to $\sim 15 \mu \mathrm{m}$ in diameter.

For the example shown in Fig. 5, the growth in the droplet distribution is unlikely to induce precipitation formation while the parcel is still in the chimney. Precipitation formation remains an active area of research, but it is generally believed that the coalescence processes that control drizzle formation are very slow until at least some of the droplets grow to 25-30 $\mu \mathrm{m}$ in diameter [18]. However, the droplet size distribution is strongly influenced by the initial particle size distribution in addition to the rate of water condensation. For example, if the particle concentrations were smaller by a factor of four with the same size distribution, then the same liquid water mixing ratio would lead to a $60 \%$ increase in droplet diameters. Such an occurrence is not unrealistic, and the larger droplets that would thereby result would be at the lower edge of the range where precipitation formation is thought to become likely. It may also be possible for the presence of a very small number of very large particles (so-called "giant CCN") to induce drizzle formation. Even if precipitation formation does not occur, cloud formation in the chimney could lead to practical complications- if water is condensing on particles within the chimney, it is 
also likely to be condensing on the chimney structure. In that event the condensed water will tend to flow downward toward the turbines and collector, presumably with negative impact.

\section{Parcel simulations with entrainment}

Having demonstrated that cloud formation is likely to occur and that even precipitation is possible within the solar chimney when significant water vapor has been added to the parcel, the next question is what happens to parcels with lower water vapor loadings after they exit the chimney. As has been noted, the adiabatic assumption is expected to break down once the parcel exits the chimney. Simulating parcel development outside the outside the chimney requires that entrainment processes be included. Thus, the newly modified parcel model was used to evaluate how the parcels in the "LoRH" and "No Enhancement" cases develop after exiting the solar chimney. As discussed in section 2.3, the full set of simulations were performed using a constant entrainment rate of $30 \% \mathrm{~km}^{-1}$ once the hypothetical chimney height of $1000 \mathrm{~m}$ has been reached, and then repeated at a higher entrainment rate of $30 \%$ per $100 \mathrm{~m}$; these simulations covered the presumed range of likely entrainment rates. No entrainment simulations were conducted for the "HiRH" cases, since the advanced cloud development within the chimney makes those conditions unviable for solar chimney power plants. It should also be noted that while the inclusion of entrainment does improve the realism of the model, it is still somewhat idealized- the parcel updraft velocity remains constant throughout the entrainment simulations, rather than decreasing as less buoyant external air is mixed in. The results are best interpreted as indications of the potential for cloud development, rather than as detailed characterizations of the clouds themselves.

Figure 6 presents vertical profiles of temperature, supersaturation, and water content for one of these new simulations, demonstrating the effect of entrainment on a rising parcel. As in Fig. 2, these profiles are from the October "LoRH" simulation, with $\Delta T=30 \mathrm{~K}$ and $w=15 \mathrm{~m} \mathrm{~s}^{-1}$; the only difference in the initialization parameters is the inclusion of entrainment $\left(30 \% \mathrm{~km}^{-1}\right)$ starting at the $1000 \mathrm{~m}$ level. For easy comparison, the traces from Fig. 2 are included again in Fig. 6. As would be expected, there are no differences below $1000 \mathrm{~m}$ between the adiabatic and entrainment simulations, but above that height the entrainment of cooler, drier air into the rising air mass substantially affects cloud development within the parcel. Above the $1000 \mathrm{~m}$ level, the trends for temperature and water vapor and total water mixing ratios immediately diverge from the trends observed during the adiabatic simulations. The temperature profile (Fig. 6a) decreases somewhat more rapidly as the adiabatic cooling is enhanced by the in-mixing of ambient air. The effect on the water profile is more pronounced (Fig. 6c), because there is a much larger difference in water 
vapor content between the parcel and its surroundings and because water content was conserved in the adiabatic simulations (whereas temperature still decreased). The combined effects of entrainment on temperature and water content are visible in Fig. 6b: it delays the onset of supersaturated conditions (cloud base occurs at 3400 versus $2700 \mathrm{~m}$ ), and reduces the magnitude of the maximum supersaturation reached by the parcel (approximately $0.41 \%$ versus $0.70 \%$ ). The conversion of water vapor to liquid water is also severely inhibited. In the adiabatic case, the liquid water content of the parcel increased continuously once droplet activation occurred; a maximum of $1.3 \times 10^{-2} \mathrm{~kg} / \mathrm{kg}$ was reached by the end of the simulation. In contrast, when entrainment is included the liquid water content increases for a period, reaching a maximum value of $6.1 \times 10^{-4} \mathrm{~kg} / \mathrm{kg}$, but then begins to decrease again and is essentially zero by the end of the simulation. This is a direct result of the entrainment process- the continuous mixing in of drier air causes supersaturation to decrease more rapidly than in the adiabatic case, and eventually the parcel becomes subsaturated and the cloud begins to evaporate. The details of the evaporation stage are not displayed in Figure 5 to avoid confusion, since the assumptions in the parcel model are most reliable for the early stages of cloud development.

The impact of entrainment on the evolution of the cloud droplet distribution is presented in Figure 7. As in the adiabatic case (Fig. 5), the size distribution changes very little before the parcel becomes saturated. At the saturation point, at approximately $3400 \mathrm{~m}$ in Fig. 7, the droplets begin to activate and grow rapidly. The mode diameter reaches approximately $7 \mu \mathrm{m}$ by the time the parcel reaches $4000 \mathrm{~m}$; above this height, there continues to be some growth at the upper end of the droplet size distribution, but the mode diameter stays relatively constant. Though not shown, eventually entraining dry air causes the water vapor mixing ratio to become subsaturated again and the cloud begins to evaporate. This growth / stability / evaporation cycle is in strong contrast with the behavior predicted by the adiabatic simulations, wherein water condensed continually onto the available droplets after activation occurred, leading to much more liquid water, a larger mode droplet diameter, and no cloud top. In sum, the inclusion of entrainment greatly inhibits cloud development within the rising parcel.

The results of all of the "LoRH" simulations with entrainment are summarized in Figure 8. Many of the trends in the results are similar to those observed in the adiabatic simulations and discussed in Section 3. As before, the maximum liquid water content is relatively insensitive to the updraft velocity and generally increases with increasing $\Delta T$. The maximum supersaturation exhibits a stronger dependence on updraft velocity, and a weaker dependence on $\Delta T$. However, there are also important differences. Most importantly, the results indicate that for several of the cases, the 
parcel never becomes supersaturated and no cloud forms. This is true for all of the January simulations, one of the October simulations, and all of the "NoEnhancement" simulations (not shown). In the lone October case for which cloud formation does not occur, the maximum saturation ratio is very close to one; it's likely that a very small change in the initialization conditions would lead to cloud formation. The January and "NoEnhancement" results are much more robust. For the January simulations the maximum parcel relative humidity never exceeds $97 \%$, and usually peaks between $65 \%$ and $90 \%$. The lack of cloud formation is due to the relative lack of water vapor available for condensation in the January simulations relative to the other seasons (October is the next driest season). In the adiabatic simulations this relative lack of water resulted in higher cloud bases; when entrainment is included this already limited supply of water is diluted before the parcel approaches the saturation point.

The results in Figures 6-8 are very sensitive to the choice of entrainment rate. This is not surprising- increasing entrainment from zero to $30 \% \mathrm{~km}^{-1}$ results in the differences already described in this and the previous section, including notably the absence of any cloud formation during the January seasonal representation. Increasing the entrainment again by an order of magnitude (to $30 \%$ per $100 \mathrm{~m}$ ) severely impacts the cloud forming potential of the rising parcels, such that only the five "LoRH" simulations for the July seasonal representation with $\Delta T=40 \mathrm{~K}$ resulted in any cloud. Moreover, these five clouds were less than $300 \mathrm{~m}$ thick and had liquid water contents of $3.5 \times 10^{-4} \mathrm{~kg} \mathrm{~kg}^{-1}$ or below (not shown). Such a severe sensitivity to the entrainment rate, which depends primarily on the external environmental conditions, prevents a conclusive assessment of the cloud development potential in the absence of more detailed climatological data.

\section{Implications for Facility Design}

The results of the adiabatic and entrainment-affected cloud parcel simulations indicate that for standard operating conditions, moderate to high water vapor enhancement in a solar chimney power facility can lead to cloud formation either within the chimney itself or in the plume after it exits the chimney. Cloud formation within the chimney is certainly an outcome to be avoided, as a practical matter. While most of the excess water vapor would condense onto the available particles, some fraction would also condense onto the walls of the chimney and perhaps then flow downward toward the turbines. The implications of this would depend on the specific design of the power plant. There are certainly ways to remove the wall condensate from the system, but these would inevitably lead to higher construction and maintenance costs and would likely also influence chimney efficiency. On the other hand, in the absence of mitigation effects water may 
flow downward along the chimney walls, and could even initiate a feedback mechanism causing the cloud base height to be lowered further. Fortunately, the simulations results indicate that cloud formation within the chimney occurs only when very large quantities of water vapor are added to the parcel before it enters the chimney; it should not be difficult to avoid operating conditions that would lead to this result.

Cloud formation in the plume after the parcel has left the chimney is more difficult to predict. This is true particularly because of the severe sensitivity to the entrainment rate. In the absence of entrainment, cloud formation always occurred. When the entrainment rate was $30 \%$ per 1000 $\mathrm{m}$, cloud formed in nearly all of the simulations, except during driest season (January). When the entrainment rate was increased by a factor of ten, only the hottest, wettest parcels resulted in any cloud development. Clearly the formation of a plume would depend strongly on the external environment as well as on the chimney conditions.

In the absence of cloud development, the presence of excess water in the chimney plume would have a negligible impact on plant performance beyond the thermodynamic effects noted previously be Kröger and Blaine [8] and Ninic [9]. The impact when cloud does form depends on the specifics of development, which again depend on the external environment. The presence of a persistent, thick cloud in the chimney plume would block some fraction of the incident solar radiation from reaching the facility's collector, and would thereby cause a reduction in overall plant capacity. A full evaluation of the phenomenon is beyond the scope of this study, but it is possible to derive a rough upper limit estimate based on the simulations results presented here. We start by calculating what fraction of the solar collector would be shaded by the plume. At the top of the chimney, the plume can be assumed to be the width of the chimney, but it will expand as it moves downwind. We need to determine how much it expands, which requires a few simplifying assumptions. First, if we assume that the wind speed above the chimney is $10 \mathrm{~m} \mathrm{~s}^{-1}$ and recall that the diameter of the collector is $7000 \mathrm{~m}$, we find that the parcel will remain over the collector for $350 \mathrm{~s}$ after it exits the top of the chimney. A parcel with an updraft velocity of $15 \mathrm{~m}$ $\mathrm{s}^{-1}$ will therefore rise $5250 \mathrm{~m}$ before passing over the edge of the collector. Using this rise distance and the $30 \% \mathrm{~km}^{-1}$ (two-dimensional) entrainment rate, a simple mass balance argument indicates that the width of the parcel will expand by a factor of two by the time it reaches the edge of the collector (for every $1000 \mathrm{~m}$ of plume rise, the amount of air in the parcel and hence the parcel cross section increases by $30 \% ; 1.30^{(5.25 / 2)} \approx 2.0$ ). The chimney diameter for the proposed Australian facility would be $150 \mathrm{~m}$, so the plume would be $300 \mathrm{~m}$ wide when it crosses the collector edge. Since the collector's circumference is $\sim 22000 \mathrm{~m}$, the wedge of the plume would 
cast a shadow over $\sim 1.4 \%$ of the collector's area. While the solar collector can operate with both direct and diffuse solar radiation, if we assume the limiting case of complete cloud opacity, this would lead to an equivalent $1.4 \%$ reduction in incident radiation, and thus plant capacity.

While it does not seem large, a periodic $1.4 \%$ reduction in plant capacity due to the presence of cloud would affect somewhat the profitability of the plant. However, the impact of cloud formation would not occur in isolation, and it may be that the costs associated with excess water vapor and potential cloud formation would be offset by other gains. This is certainly a possibility if the water is added to the system to increase the potential energy of the parcel at the base of the chimney, as has been suggested by Ninic [9]. It may also be true that the gains realized by using the collector area for agriculture or by incorporating water-filled heat storage devices would outweigh the costs associated with evaporative losses. In these latter circumstances it is likely the careful design would prevent most of the potential loss, and that cloud formation could be avoided. In any event, a full economic analysis of the costs and benefits associated with excess water in a solar chimney power facility would depend on the details of the final design and is beyond the scope of this work.

\section{Conclusions}

The simulations performed for this work indicate that excess water vapor has the potential in some circumstances to adversely affect the performance of a large solar chimney power generation facility. For very high water vapor concentrations, cloud would probably form inside the chimney itself, with precipitation formation possible in some cases. The end result of inchimney condensations is likely to be liquid water falling to ground level at the base of the chimney. For more moderate water vapor enhancements, the potential for cloud formation varied seasonally and was very sensitive to the assumed entrainment rate. In many cases no cloud formed and thus there were no negative impacts of the excess water vapor. However, in several cases there was cloud formation in the plume after it exited the chimney. These clouds would likely not precipitate but could persist long enough to affect plant performance. A rough upper limit estimate indicates that when clouds form, they could cast a shadow covering as much as $1.4 \%$ of the solar collector, resulting in a reduction of incident radiation for the facility and thus a reduced power generation capacity.

It is clear from the results here that the potential for cloud formation associated with solar chimney power plants is such that the effect should be considered in detail in plant design and site selection. This should include more detailed analyses of the interactions between the expected water vapor inputs, chimney dynamics, and the local climatology. From the results presented 
here, it is certainly possible that no cloud would form and thus that the overall impact of excess water vapor would be negligible. If cloud does form, then the associated negative impacts would have to be weighed against any gains realized by the presence of water in the system .

\section{Acknowledgements}

This work was completed primarily at the National Center for Atmospheric Research with support there for Dr. VanReken from the Advanced Study Program. NCAR is sponsored by the National Science Foundation. Dr. Nenes acknowledges support from a Blanchard-Milliken Young Faculty Fellowship. The authors would also like to thank the anonymous reviewers, whose helpful comments led to an improved manuscript. 


\section{References}

[1] Schlaich, J., Bergermann, R., Schiel, W., and Weinrebe, G., 2005, "Design of Commercial Solar Updraft Tower Systems - Utilization of Solar Induced Convective Flows for Power Generation,” ASME J. Solar Energy Eng., 127(1), pp. 117-124.

[2] Haaf, W., Friedrich, K., Mayr, G., and Schlaich, J., 1983, "Solar Chimneys Part I: Principle and Construction of the Pilot Plant in Manzanares," Int. J. Solar Energy, 2, pp. 3-20.

[3] Haaf, W., 1984, "Solar Chimneys Part II: Preliminary Test Results from the Manzanares Pilot Plant,” Int. J. Solar Energy, 2, pp. 141-161.

[4] Gannon, A. J., and von Backström, T. W., 2000, "Solar Chimney Cycle Analysis with System Loss and Solar Collector Performance," ASME J. Solar Energy Eng., 122(3), pp. 133-137.

[5] Bernardes, M. A. D. S., Voß, A., Weinrebe, G., 2003, "Thermal and Technical Analyses of Solar Chimneys," Solar Energy, 75(6), pp. 511-524.

[6] Dai, Y. J., Huang, H. B., Wang, R. Z., 2003, "Case Study of Solar Chimney Power Plants in Northwestern Regions of China," Renewable Energy, 28(8), pp. 1295-1304.

[7] EnviroMission, Ltd., 2005, Annual Report, ACN 094963238.

[8] Kröger, D. G., and Blaine, D., 1999, "Analysis of the Driving Potential of a Solar Chimey Power Plant,” R\&D Journal, 15(3), 85-94.

[9] Ninic, N., 2006, "Available Energy of the Air in Solar Chimneys and the Possibility of its Ground-Level Concentration," Solar Energy, 80, pp. 804-811.

[10] Nenes, A., Ghan, S., Abdul-Razzak, H., Chuang, P. Y., and Seinfeld, J. H., 2001, "Kinetic Limitation on Cloud Droplet Formation and Impact on Cloud Albedo," Tellus Ser. B, 53, pp. 133-149.

[11] Pruppacher, H. R., and Klett, J. D., 1997, Microphysics of clouds and precipitation. Kluwer, Dordrecht, Chap. 12.

[12] Seinfeld, J. H., and Pandis, S. N., 1998, Atmospheric Chemistry and Physics, John Wiley, New York, Chap. 15.

[13] Nenes, A., Charlson, R. J., Facchini, M. C., Kulmala, M., Laaksonen, A., and Seinfeld, J. H., 2002, "Can Chemical Effects on Cloud Droplet Number Rival the First Indirect Effect?," Geophys. Res. Lett., 24(17), 1848, doi:10.1029/2002GL015295.

[14] Rissman, T. A., Nenes, A., and Seinfeld, J. H., 2004, "Chemical Amplification (or Dampening) of the Twomey Effect: Condition Derived from Droplet Activation Theory," J. Atmos. Sci., 61(8), pp. 919-930.

[15] Lance, S., Nenes, A., and Rissman, T. A., 2004, "Chemical and Dynamical Effects on Cloud Droplet Number: Implications for Estimates of the Aerosol Indirect Effect," J. Geophys. Res., 109, D22209, doi:10.1029/2004JD004596.

[16] Jensen, M. P., and Del Genio, A. D., 2006, "Factors Limiting Convective Cloud-top Height at the ARM Nauru Island Climate Research Facility," J. Climate, 19(10), pp. 2105-2117.

[17] Jaenicke, R., 1998, "Atmospheric Aerosol Size Distribution," Atmospheric Particles, Harrison, R. M., and van Griecken, R. E. (Eds.), John Wiley \& Sons, New York, pp. 1-29.

[18] Young, K. C., 1993, Microphysical Processes in Clouds, Oxford University Press, New York, Chap. 9. 


\section{Tables}

Table 1. Seasonal Representation for Cloud Parcel Simulations.

\begin{tabular}{|l|c|c|c|c|}
\hline & January & April & July & October \\
\hline \hline Temperature (K) & 304.7 & 296.3 & 288.2 & 296.6 \\
Relative Humidity: & & & & \\
"LoRH" Cases & $25 \%$ & $40 \%$ & $54 \%$ & $33 \%$ \\
"HiRH" Cases & $80 \%$ & $80 \%$ & $80 \%$ & $80 \%$ \\
No Enhancement & $5.4 \%$ & $7.9 \%$ & $9.6 \%$ & $6.5 \%$ \\
\hline
\end{tabular}

Table 2. Initialization Parameters for Cloud Parcel Model Simulations.

\begin{tabular}{|l|l|}
\hline Parameter & Values \\
\hline \hline Pressure & $9 \times 10^{4} \mathrm{~Pa}$ \\
Mass uptake coefficient & 1.0 \\
Cloud depth & $5000 \mathrm{~m}$ \\
Number of time steps & 1000 \\
Liquid water content & $0.0 \mathrm{~kg} \mathrm{~kg}^{-1}$ \\
Temperature increase $(\Delta T)$ & $15,25,30,35,40 \mathrm{~K}$ \\
Vertical velocity $(w)$ & $1,5,10,15,20 \mathrm{~m} \mathrm{~s}^{-1}$ \\
\cline { 2 - 2 } Entrainment rate & $30 \%, 300 \% \mathrm{~km}^{-1}$ \\
\hline
\end{tabular}

${ }^{a}$ The cloud depth parameter determines how long the simulation continues beyond the initial point of water condensation.

Table 3. Model lognormal aerosol populations.

\begin{tabular}{|l|c|c|c|c|c|}
\hline Mode & $N\left(\mathrm{~cm}^{-3}\right)^{\mathrm{a}}$ & $D_{p}(\mu \mathrm{m})^{\mathrm{a}}$ & $\sigma^{\mathrm{a}}$ & $\begin{array}{c}\text { Soluble } \\
\text { Fraction }^{\mathrm{b}}\end{array}$ & $\begin{array}{c}\text { Insoluble } \\
\text { Fraction }\end{array}$ \\
\hline Nuclei & 3200 & 0.020 & 1.45 & 0.40 & 0.60 \\
Accumulation & 2900 & 0.116 & 1.65 & 0.75 & 0.25 \\
Coarse & 0.3 & 1.8 & 2.40 & 0.10 & 0.90 \\
\hline
\end{tabular}

${ }^{a}$ See text for parameter definitions.

${ }^{b}$ The soluble fraction is assumed to consist entirely of ammonium sulfate. 


\section{Figure Captions}

Figure 1. Schematic of a solar chimney power generation facility. Air enters the solar collector (a) at ambient temperature and relative humidity. As air passes through the collector (b), it is heated by solar radiation and its water vapor content may also increase for reasons described in the text. At (c), the parcel passes through a turbine, where part of the kinetic energy is converted to electricity. The parcel, driven by buoyancy, then proceeds up the chimney (d).

Figure 2. Typical vertical profiles from an adiabatic cloud parcel simulation. The results here are taken from a "LoRH" simulation for the October seasonal representation, with $w=15$ $\mathrm{m} \mathrm{s}^{-1}$ and $\Delta T=30 \mathrm{~K}$. (a) The temperature decreases as the parcel rises, with a change in the rate of decrease at the onset of condensation. (b) The supersaturation profile. As the parcel cools it surpasses its saturation point and condensation begins. The peak supersaturation occurs at cloud base. (c) Water mixing ratios. The water vapor mixing ratio is the short dashed trace, the liquid water mixing ratio is the long dashed trace, and the total water is solid. The liquid water mixing ratio is zero until cloud base is reached, and total water is conserved save for a minor numerical drift at the start of the simulation. In the figure, the solid black horizontal line indicates the level of the chimney top $(1000 \mathrm{~m})$ and the dashed black horizontal line indicates the cloud base level at approximately $2700 \mathrm{~m}$.

Figure 3. Results from the adiabatic "LoRH" simulations for each of the four seasonal representations. The data are grouped along the bottom axis by temperature increase. Within each group, the updraft velocities $\left(1,5,10,15\right.$, and $\left.20 \mathrm{~m} \mathrm{~s}^{-1}\right)$ increase from left to right for all data. Cloud base heights are indicated by triangles in the top graph for each season; the chimney top is indicated by the dashed line. The lower graph for each season includes the final liquid water content (bars) and the maximum supersaturation (squares, with the saturation point indicated by a horizontal dotted line).

Figure 4. Same as Figure 3, but for the "HiRH" adiabatic simulations.

Figure 5. Evolution of the cloud droplet distribution during a "HiRH" simulation. This example is from the October seasonal representation, with $w=15 \mathrm{~m} \mathrm{~s}^{-1}$ and $\Delta T=30 \mathrm{~K}$. In this example, cloud formation begins at a height of $\sim 600 \mathrm{~m}$, and the chimney top is indicated by a dashed line. 
Figure 6. Vertical profiles from cloud parcel simulation with entrainment starting at $1000 \mathrm{~m}$ (the chimney top height). The entrainment rate is $30 \% \mathrm{~km}^{-1}$. As in Figure 2, the results here are taken from a "LoRH" simulation for the October seasonal representation, with $w=15 \mathrm{~m} \mathrm{~s}^{-1}$ and $\Delta T=30 \mathrm{~K}$. Plots (a)-(c) are as in Figure 2. Black traces are from the entrainment simulation, and gray traces reproduce for comparison the results of the adiabatic simulations. Below the $1000 \mathrm{~m}$ chimney top height, the results of the two simulations are identical. However, above chimney top there are substantial differences between them, including a more rapidly decreasing temperature, an increase in the cloud base height, a decrease in the peak supersaturation, and drastic changes to the water mixing ratios. See the text for a more complete explanation.

Figure 7. Evolution of the cloud droplet distribution for a "LoRH" simulation with entrainment. These results are from the same simulation as Figure 6.

Figure 8. Results from the "LoRH" simulations with entrainment for each of the four seasonal representations. The grouping and panels of each graph are as in Figure 3. Note that the scale for the liquid water content has been reduced by a factor of four. Cloud formation does not occur in any of the January simulations, nor for one of the October simulations- due to entrainment the parcel never becomes supersaturated in these simulations. 
Figures

Figure 1

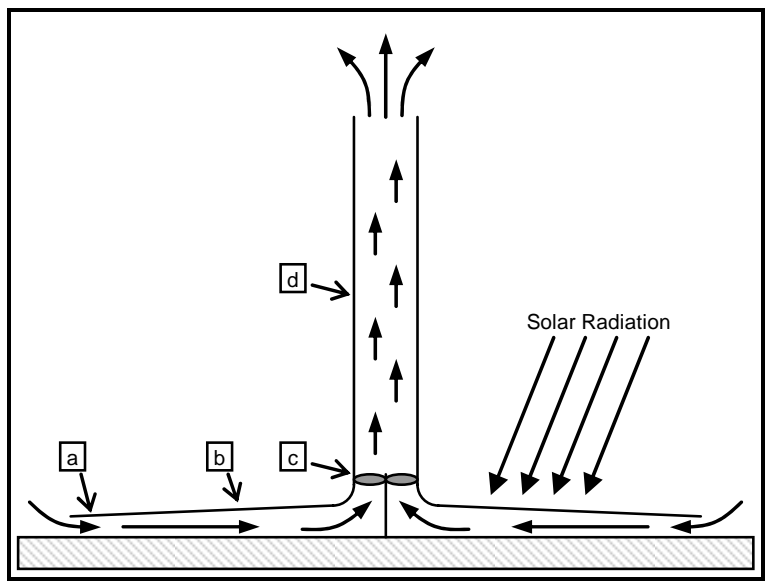

$\underline{\text { Figure } 2}$

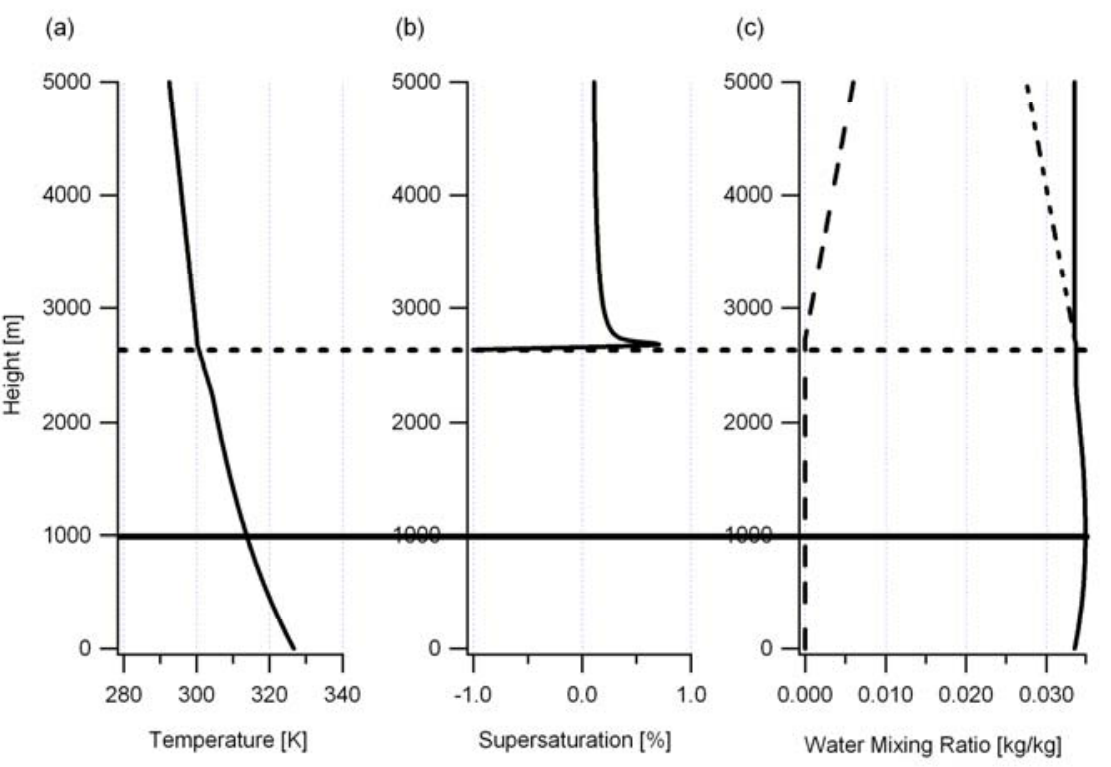


Figure 3
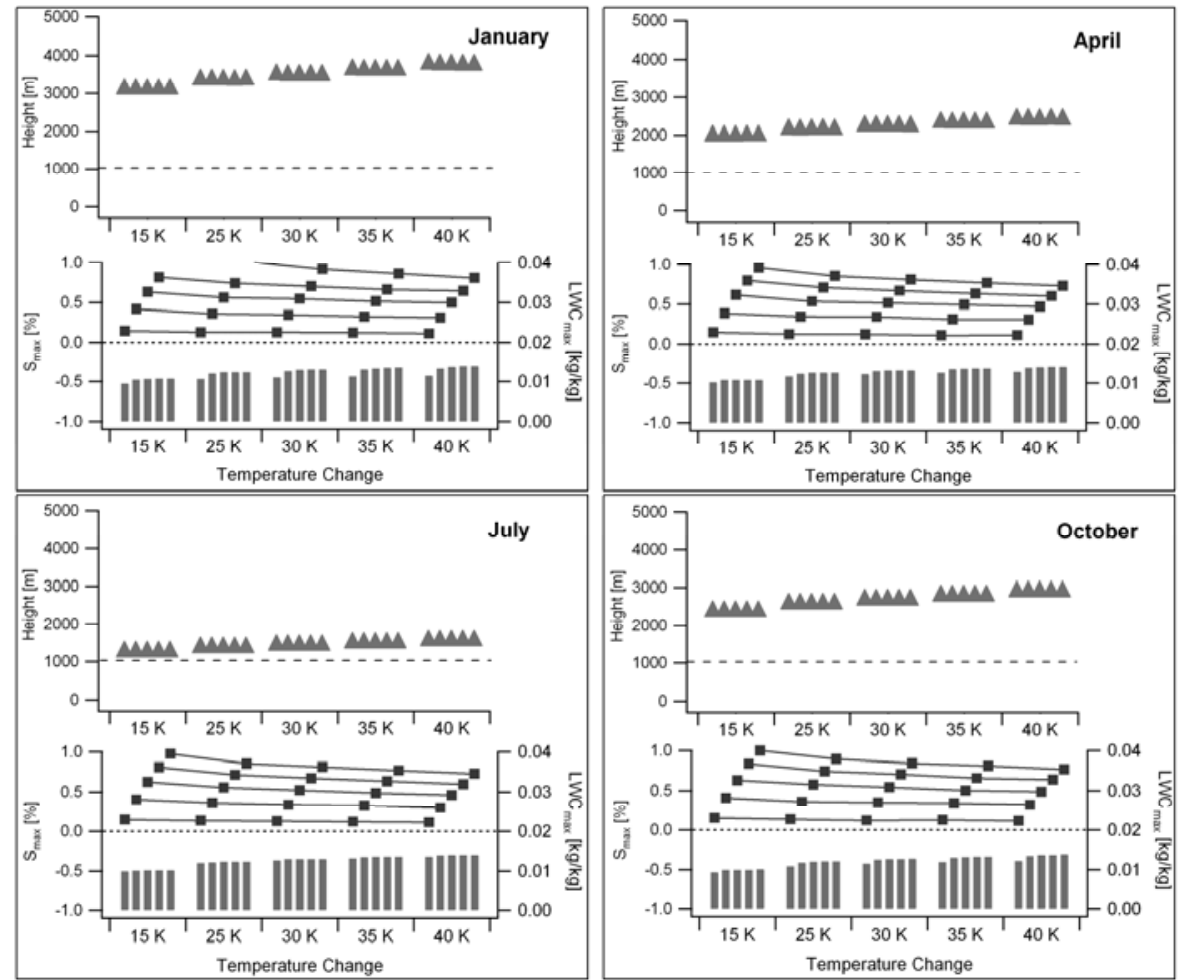

Figure 4
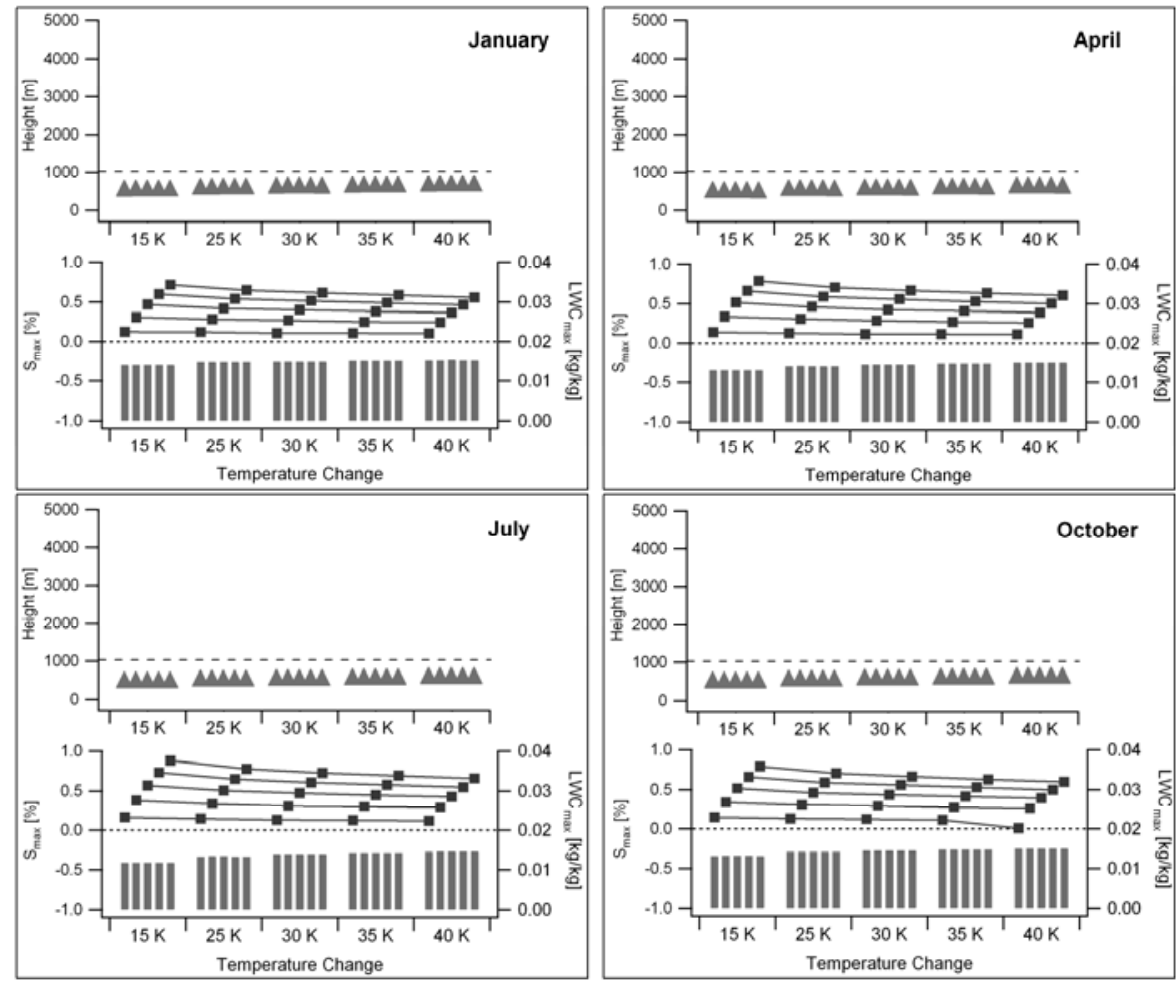
Figure 5

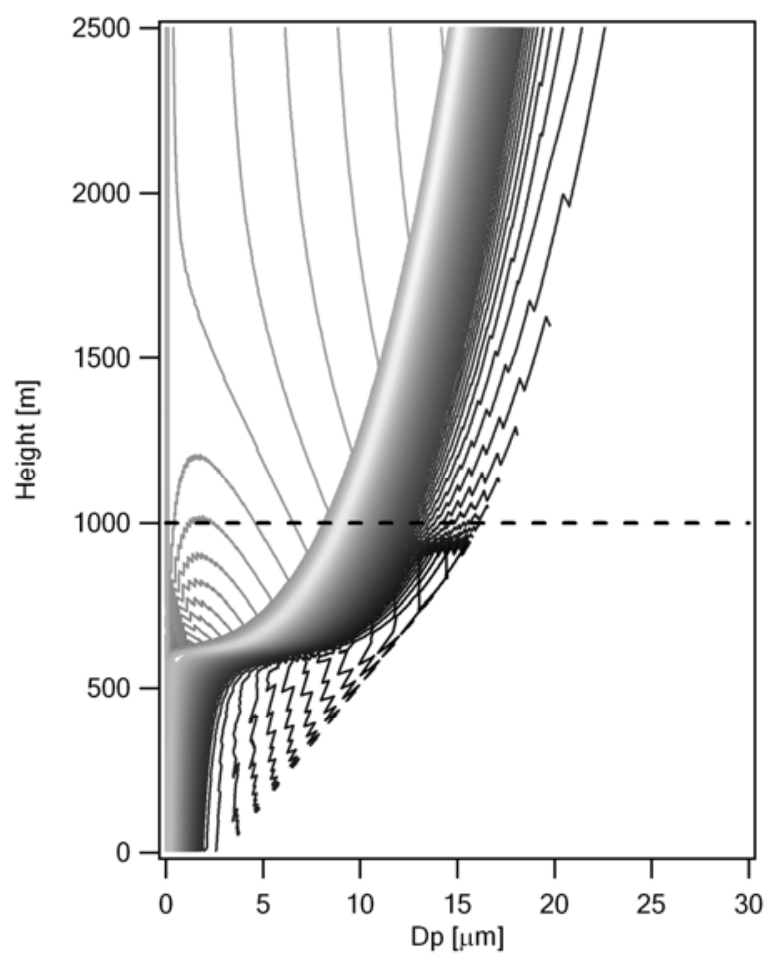

$\underline{\text { Figure } 6}$

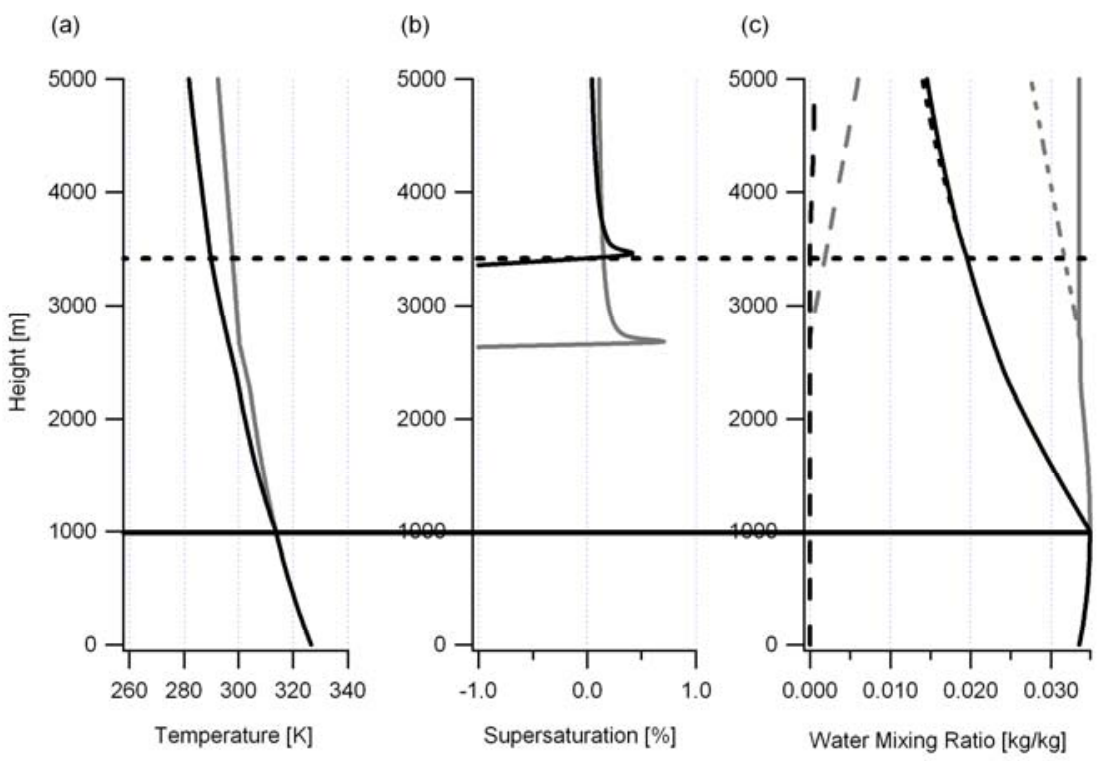


Figure 7

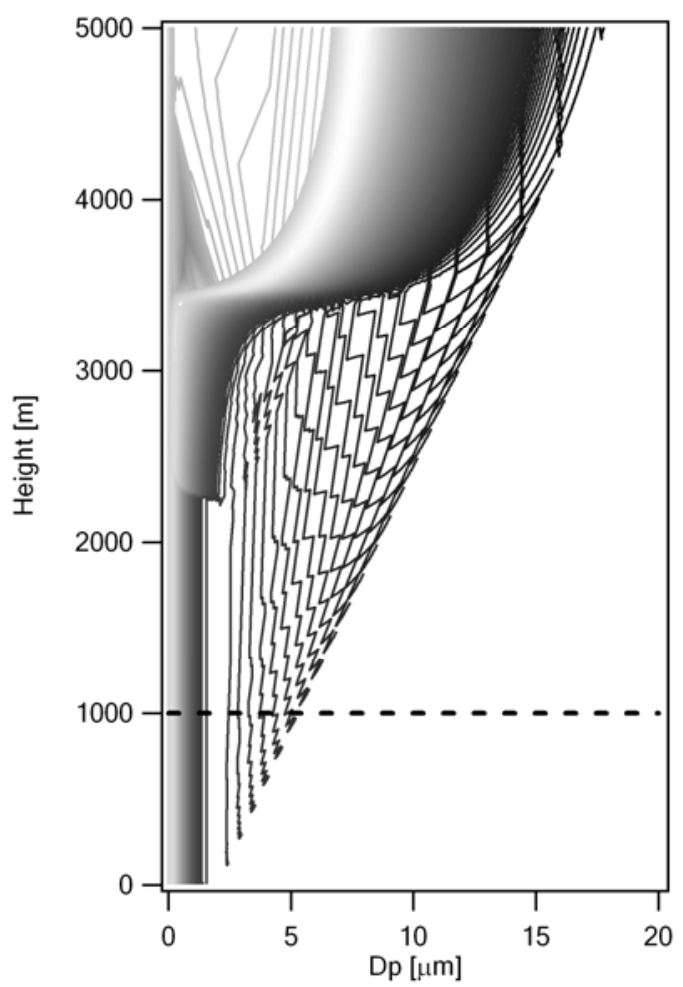

Figure 8
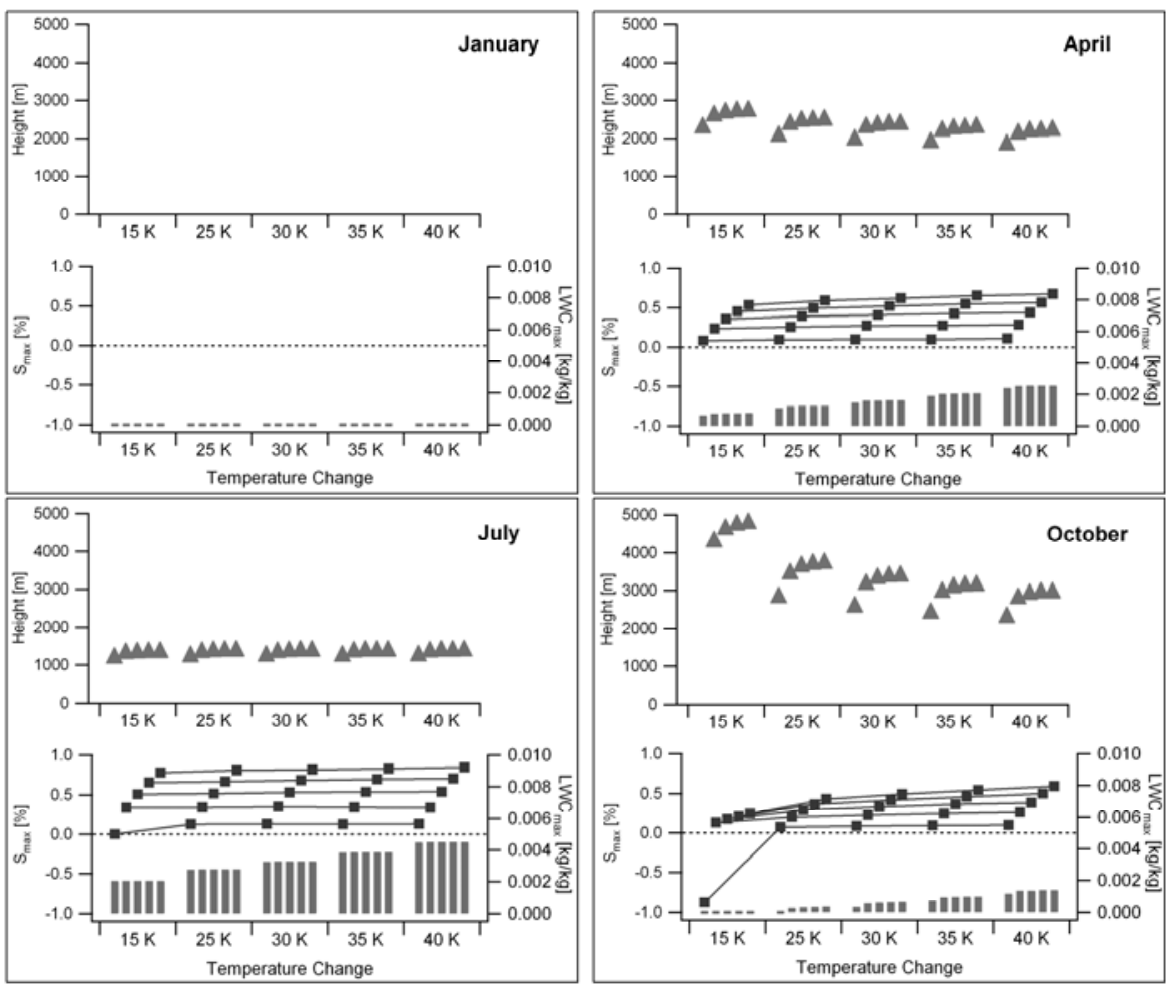